\title{
SYNTHESIS AND CHARACTERIZATION OF SILVER NANOPARTICLES FROM MARINE BROWN SEAWEED AND ITS ANTIFUNGAL EFFICIENCY AGAINST CLINICAL FUNGAL PATHOGENS
}

\author{
RAJESHKUMAR $\mathbf{S}^{1 *}$, MALARKODI $\mathrm{C}^{2}$, VENKAT KUMAR $\mathbf{S}^{1}$ \\ School of Bio-Sciences and Technology, VIT University, Vellore, Tamil Nadu, India. ${ }^{2}$ Department of Chemistry, University of Delhi, \\ Delhi, India. Email: ssrajeshkumar@hotmail.com
}

Received: 09 September 2016, Revised and Accepted: 27 October 2016

\section{ABSTRACT}

Objectives: The aim of this study is to synthesize silver nanoparticles using the algal extract of Padina tetrastromatica and evaluate its antifungal activity against pathogenic fungus isolated from clinical samples.

Methods: Dried algal biomass was used to prepare the pure algal extract and added with $1 \mathrm{mM} \mathrm{AgNO}_{3}$, and the color change was noted and recorded by ultraviolet (UV)-vis spectrophotometer. The morphological characteristics were analyzed by scanning electron microscopy (SEM) and transmission electron microscopy (TEM). Crystalline structure was analyzed by SAED pattern. Antifungal activity was performed by agar well diffusion method against various pathogenic fungi.

Results: Formation of brown color at 15 minutes indicates the production of silver nanoparticles by the extract of brown algae $P$. tetrastromatica. Surface plasmon resonance band was centered at $440 \mathrm{~nm}$ which was observed by UV-vis spectrophotometer. SEM image revealed spherical and cubical nanoparticles with high agglomeration, and energy-dispersive X-ray illustrates elemental components of silver formed at $3 \mathrm{keV}$. TEM shows spherical, truncated, and ellipsoidal nanoparticles and also it evidences the algae compounds that are capped with nanoparticles. SAED pattern proved four diffraction face-centered cubic rings at (111), (200), (220), and (311) which indicates the crystalline nature of nanoparticles. Silver nanoparticles show high inhibition activity against Fusarium sp, Aspergillus niger, Candida albicans, Aspergillus fumigatus, and Aspergillus flavus at different concentrations. P. tetrastromatica-mediated synthesis of silver nanoparticles shows rapid and eco-friendly silver ion reduction process.

Conclusion: Therefore, this present study elucidates that algae-mediated synthesized silver nanoparticles have antifungal activity against pathogenic fungi, so it can be developed as a novel medicine for human welfare in biomedical applications in the near future.

Keywords: Padina tetrastromatica, Silver nanoparticles, Transmission electron microscopy, Antifungal activity, Green synthesis.

(c) 2017 The Authors. Published by Innovare Academic Sciences Pvt Ltd. This is an open access article under the CC BY license (http://creativecommons. org/licenses/by/4. 0/) DOI: http://dx.doi.org/10.22159/ajpcr.2017.v10i2.15127

\section{INTRODUCTION}

Manufacturing of nanomaterials is one of the most demanding and highest increasing sectors of nanotechnology. Nanotechnology in biomedical sciences receives the possibility of a wide variety of medical uses at molecular and cellular levels [1]. Biocompatible metal nanoparticles play an important role in the biomedical applications due to their optical properties such as surface plasmon resonance (SPR) and fluorescence, which is used for bioimaging and biosensing [2]. Among the noble metals, silver in colloidal state exhibits distinctive properties such as conductivity, chemical stability, catalytic, and good antibacterial activity [3]. Reduced silver ions are stable and colloidal dispersion in water or organic solvents [4]. As a natural material, silver is known to be safe to human and produces little-to-no allergic reactions when tested for curing various diseases. Physical, chemical, and biological methods are available for nanoparticle synthesis. Although the physical and chemical methods may effectively synthesize pure, well-defined nanoparticles, these methods are expensive and potentially toxic to the environment. Use of biological organisms such as bacteria, fungi, yeast, plant extract or plant biomass, and algae extract or biomass could be an alternative to these methods for the synthesis of nanoparticles in an eco-friendly manner, less time consuming, and low cost [5]. Green synthesis methods reduce the hazardous waste in the context of global efforts [6]. Nanoparticles synthesized using marine algal seaweed were quite stable in solution [7], eco-friendly, and exploited to a vast extent because the seaweeds are widely distributed, easily available, and safe to handle with a range of metabolites. Moreover, the proposed green synthesis method is an advance of bioscience, high yielding, low-cost technology, and non-toxic to vertebrate animals.
Seaweeds are the sustainable resources in the marine ecosystem which are used as food, feed, and medicine [8]. Padina tetrastromatica is marine brown algae classified under Phaeophyceae class and the family Dictyotaceae and found abundantly in the Indian coastal area, especially on the east coast, and knowledge on its bioactivities is poorly known. P. tetrastomatica possess strong anti-HBV activities [9]. Methanolic extract of $P$. tetrastromatica has spasmogenic, antifertility, hypotensive, and antiamebic properties. Petroleum ether extracts yield fatty acids, sterols, and terpenoids [10]. Sulfated polysaccharide is one of the bioactive components in the marine seaweeds which has antiinflammatory and antioxidant effects [11]. This present investigation illustrates green synthesis of silver nanoparticles using the extract of $P$. tetrastromatica and characterized by ultraviolet (UV)-vis spectrophotometer. Morphological and elemental analysis was carried out by scanning electron microscopy (SEM), transmission electron microscopy (TEM), and energy-dispersive X-ray (EDX). Crystalline structure was confirmed by SAED pattern. Thus, algae-mediated synthesized silver nanoparticles show more inhibition growth of pathogenic fungi analyzed by agar well diffusion method.

\section{METHODS}

\section{Collection and preparation of algal extract}

The brown algae P. tetrastromatica was collected from Tuticorin coastal area in south Tamil Nadu, India. The marine brown seaweed containing salt materials in its surface area was thoroughly washed by using fresh tap water for several times and washed three times using distilled water which may remove metallic compounds and it was shade dried at room temperature for 10 days. The dried algal materials were crushed 
by using mortar and pestle to get the powder form and it was stored in an air-tight container. About $1 \mathrm{~g}$ of crushed algal powder was added with $100 \mathrm{ml}$ of distilled water in $250 \mathrm{ml}$ conical flask and boiled for 5-10 minutes at $60-80^{\circ} \mathrm{C}$. The crude extract was collected and stored at $4^{\circ} \mathrm{C}$ for experimental use.

\section{Green synthesis of silver nanoparticles}

In the typical synthesis of silver nanoparticles, $10 \mathrm{ml}$ of algal extract was added into $90 \mathrm{ml}$ of $1 \mathrm{mM}$ silver nitrate aqueous solution and stirred for constant mixing under room temperature. A color change of the solution was noted by visual inspection confirming the synthesis of silver nanoparticles.

\section{Purification and characterization of synthesized silver} nanoparticles

Color change of bioreduction of silver ions in aqueous solution was monitored by double beam UV-vis spectrophotometer at different wavelength region from 320 to $700 \mathrm{~nm}$ (Perkin Elmer, Singapore). The bioreduced silver ions were purified for further characterization studies by subjecting it to centrifugation at 10,000 rpm for 15 minutes. The pellet was collected and washed in sterile double-distilled water to get free of any biological molecule present in the algal extract. The purified silver nanoparticle was morphologically characterized by using the SEM and TEM. Elemental analysis and crystalline structure of nanoparticles were examined by EDX and SAED patterns, respectively.

\section{Antifungal assay of silver nanoparticles} Clinical fungal pathogens

The five fungal pathogenic strains used in the present study were isolated from clinical samples and identified from Micro Labs, Vellore, i.e., Aspergillus niger, Aspergillus flavus, Aspergillus fumigatus, Candida albicans, and Fusarium sp.

\section{Assay of antifungal activity}

The antifungal activity of the silver nanoparticles on various fungal strains was assayed by agar plug method and spore germination inhibition assay. The fungicidal effect of the silver nanoparticles could be assessed by the inhibition of mycelial growth of the fungus and is observed as a zone of inhibition near the disc or the wells.

Rose Bengal agar medium was prepared and poured on to the Petri plates. In the plates, $0.1 \mathrm{ml}$ of the above-mentioned fungal spores were aseptically spread using the sterile cotton swabs. Cavities of $5 \mathrm{~mm}$ were made and were filled with 50,100, and $150 \mu \mathrm{l}$ of colloidal silver nanoparticles. The plates were incubated at room temperature for 24$48 \mathrm{hrs}$. After incubation period, the different levels of zone of inhibition were measured. The statistical analysis of standard error was calculated using triplicates of experiments $(n=3)$.

\section{RESULTS}

\section{Visual examination}

Synthesis of silver nanoparticles was preliminarily identified by color change during exposure of algal extract into aqueous solution of silver ions. Initially, the silver nanoparticle formation occurred within 15 minutes which was identified by color change of aqueous solution that exhibited yellowish brown color and changed into deep brown color after the $24 \mathrm{hrs}$ time incubation. The color change occurred due to the excitation of SPR of nanoparticles (Fig. 1). The intensity of color change was directly proportion to the incubation time. After $24 \mathrm{hrs}$, there is no significant color change, indicating the saturation of the reaction of silver nanoparticle formation.

\section{UV-vis spectrophotometer}

UV-vis spectrophotometer analysis silver nanoparticles formation in the algal extract and silver nitrate solution mixture has been recorded as different functional time. Silver nanoparticles exhibited a single absorbance band at $440 \mathrm{~nm}$ at 15 minutes and steadily increased in intensity at 24 hrs without any shift in the peak (Fig. 2).
SEM and EDX analysis

SEM image shows the structure of silver nanoparticles (Fig. 3). It was depicted as polydispersed spherical and cuboidal nanoparticles and also the grains are highly agglomerated which form like clusters. EDX analysis was performed for the confirmation of silver nanoparticles. Fig. 4 shows the evidence of EDX analysis in the spot profile mode which

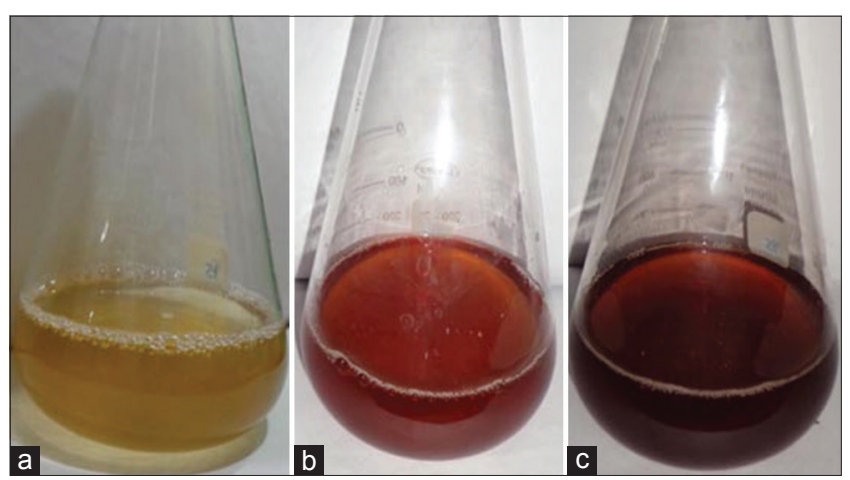

Fig. 1: Color change after the addition of algal extract with $1 \mathrm{mM}$ $\mathrm{AgNO}_{3}$ indicates formation of silver nanoparticles. (a) Initial color change, (b) 15 minutes, (c) 24 hrs

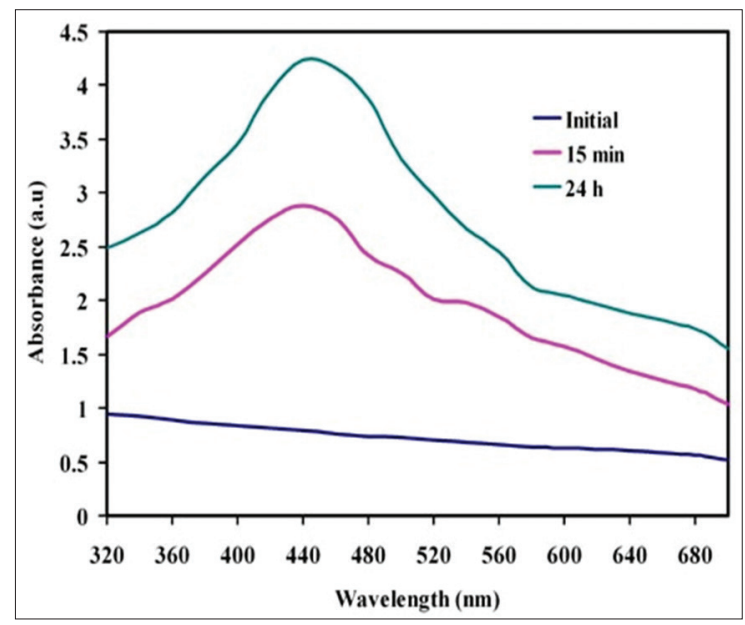

Fig. 2: Ultraviolet-visible spectra recorded the formation of nanoparticles in the reaction mixture of algal extract and $\mathrm{AgNO}_{3}$ at different time intervals showing the peak at $440 \mathrm{~nm}$

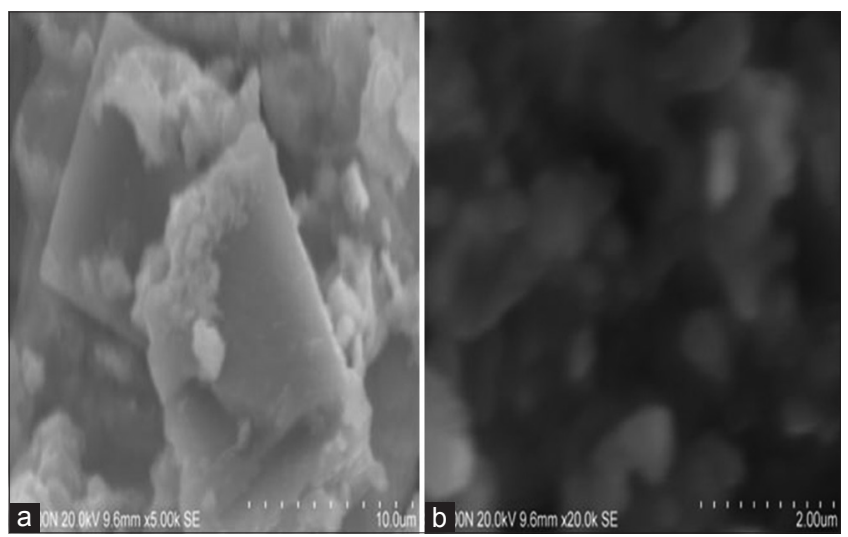

Fig. 3: Scanning electron microscope image of silver nanoparticles synthesized from Padina tetrastromatica extract showing highly agglomerated spherical-shaped nanoparticles at different magnifications 
was obtained by focusing on silver nanoparticles. The strong optical absorption band at the range of $3 \mathrm{keV}$ is observed for elemental silver. A weak signal was recorded from ' $\mathrm{O}$ ' which is due to X-ray emission from the algal extract. EDX spectrum clearly confirms the purity of the silver nanoparticles with the weight percentage of $57.93 \%$.

\section{TEM and SAED studies}

The morphology and crystallinity of $P$. tetrastromatica extractmediated synthesized silver nanoparticles were determined by TEM and SAED. The synthesized silver nanoparticles were formed spherical predominantly and some are truncated and ellipsoidal in the form of agglomerates. Some of the nanoparticles noted that the edges of the particles were smoother than the centers (marked by arrows) as shown in Fig. 5a. SAED pattern shows that green synthesized silver nanoparticles are of crystalline nature and few of them were recorded in the form of aggregates. The four diffraction ring (llll $\left.\begin{array}{lll}1 & 1 & 1\end{array}\right),\left(\begin{array}{lll}2 & 0 & 0\end{array}\right)$, (2 20 ), and (3 111 ) lattice planes could be indexed on the basis of the face-centered cubic (fcc) structure for silver (Fig. 5b).

\section{Antifungal activity}

The antifungal activity of silver nanoparticles was assayed by agar well diffusion method against human pathogenic fungi isolated from clinical samples. Table 1 shows the minimum inhibitory concentration of silver nanoparticles against clinically isolated pathogenic fungi at different concentrations. Here, the antifungal effect of silver nanoparticles depended on the concentration as increasing the concentration, the zone of inhibition was also increased. It shows more high antifungal activity due to their surface area and size of nanoparticles contacting with environment. Table 1 and Fig. 6 show the maximum zone formation of silver nanoparticles against Fusarium sp and A. niger with $20.03 \pm 0.033$ and $18.13 \pm 0.089$, respectively, followed by the minimum zone of inhibition

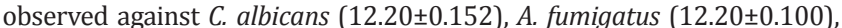
and $A$. flavus $(10.17 \pm 0.167)$ at $150 \mu$ l concentration of silver nanoparticles.

\section{DISCUSSION}

Formation of silver nanoparticles was visually identified by color change. When adding the algal extract into the silver ions solution, color was changed from pale yellow to brown at the incubation time of 15 minutes due to the excitation of SPR of nanoparticles in the reaction mixture. The deep brown color was formed after $24 \mathrm{hrs}$ of incubation; the increase in the color intensity is directly proportional to the incubation time. In this study, we have synthesized silver nanoparticles rapidly at 15 minutes using the extract of $P$. tetrastromatica. Similarly, Kumar et al. [12] synthesized silver nanoparticles within 20 minutes by using the extract of Sargassum tenerrimum and Singaravelu et al., [7] synthesized gold nanoparticles after the 24 hrs incubation time using Sargassum wightii.

UV-vis spectroscopy analysis is an important tool for optical and structural characterization of silver nanoparticles and it is an indirect method to determine the reduction of silver nitrate to silver nanoparticles in the aqueous solution. The optical property of silver nanoparticles depends mainly on size and shape [13]. There is no specific peak change in the reaction mixture as shown in UV-vis spectrophotometer. No change in peak due to the excitations of SPR of nanoparticles indicates that nanoparticles are in spherical structure which was further confirmed by

Table 1: Antifungal activity of silver nanoparticles synthesized from algal extract of Padina tetrastromatica

\begin{tabular}{llll}
\hline Pathogenic fungi & \multicolumn{3}{l}{ Zone of inhibition (mm in diameter) } \\
\cline { 2 - 4 } & $\mathbf{5 0} \boldsymbol{\mu l}$ & $\mathbf{1 0 0} \boldsymbol{\mu l}$ & $\mathbf{1 5 0} \boldsymbol{\mu l}$ \\
\hline Aspergillus niger & $13.30 \pm 0.153$ & $15.20 \pm 0.116$ & $18.13 \pm 0.089$ \\
Aspergillus flavus & $08.27 \pm 0.146$ & $08.77 \pm 0.146$ & $10.17 \pm 0.167$ \\
Aspergillus & $08.17 \pm 0.167$ & $11.07 \pm 0.120$ & $12.10 \pm 0.100$ \\
fumigatus & & & \\
Candida albicans & $10.20 \pm 0.200$ & $10.87 \pm 0.134$ & $12.20 \pm 0.152$ \\
Fusarium sp & $18.10 \pm 0.208$ & $19.23 \pm 0.186$ & $20.03 \pm 0.033$ \\
\hline
\end{tabular}

TEM image. Similarly, Shankar et al. [14] reported that geranium leafassisted silver nanoparticles formed the SPR band at $440 \mathrm{~nm}$ due to the excitation of longitudinal plasmon vibrations.

The silver nanoparticles formed mostly spherical and cubical structure with high aggregation. Similarly, Jain et al. [15] reported cubic-structured silver nanoparticles synthesized by papaya fruit extract and Geoprincy et al. [16] reported aggregated nanoclustered silver nanoparticles synthesized by chemical route. Santhoshkumar

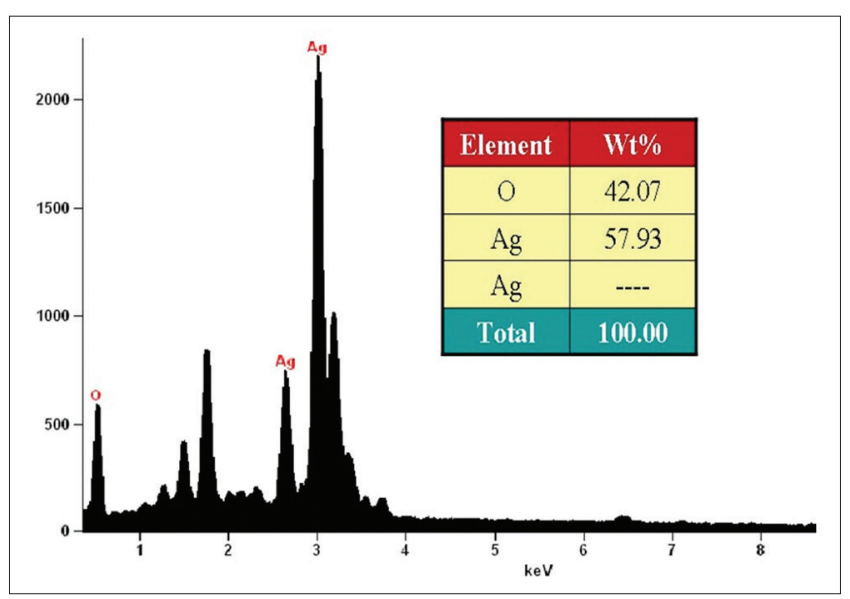

Fig. 4: Energy-dispersive X-ray analysis of Padina tetrastromaticamediated synthesized silver nanoparticles

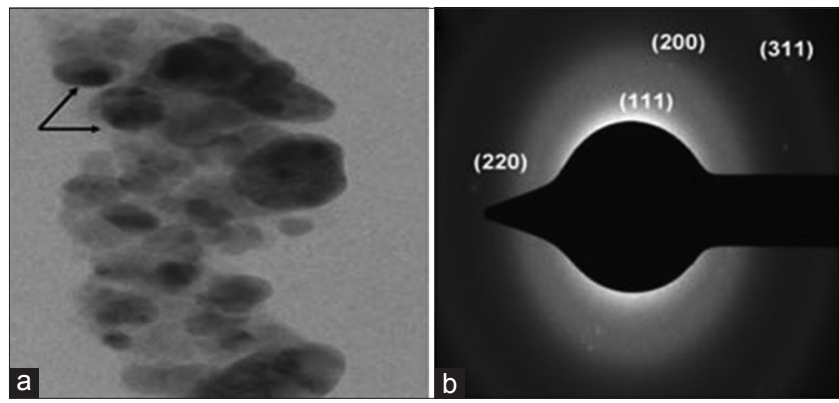

Fig. 5: (a) Transmission electron microscopy image of silver nanoparticles shows polydispersed synthesized silver nanoparticles, (b) SAED pattern of silver nanoparticles shows crystalline structure

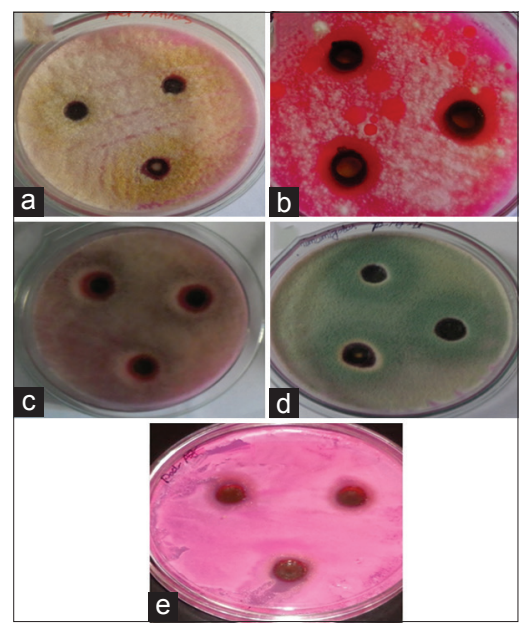

Fig. 6: Antifungal activity of silver nanoparticles synthesized against (a) Aspergillus flavus, (b) Fusarium sp., (c) Aspergillus niger, (d) Aspergillus fumigates, and (e) Candida albicans 
et al. [17] synthesized clearly distinguishable silver nanoparticles using the leaf extract of Nelumbo nucifera. Formation of elemental silver was confirmed through EDX analysis at the binding energy of $3 \mathrm{keV}$; it is the best evidence to identify that formation of pure silver.

Truncated, spherical, and ellipsoidal nanoparticles were identified by TEM. The edges of nanoparticles were smoother than the center which indicates that the proteins in algal extract may cap the silver nanoparticles Similarly, Ahmad et al. [18] suggested that proteins in basil plant are present among the particles and adhered to their surfaces. SAED shows four diffraction rings of fcc for silver. SAED report clearly indicates that synthesized silver nanoparticles using algal extract of $P$. tetrastromatica are of crystalline structure.

Among the noble metal nanoparticles, silver nanoparticles have been widely used for many applications. In this study, the antifungal activity of silver nanoparticles was evaluated. The antimicrobial activity of silver nanoparticles mainly depends on the size and shape of the nanoparticles. The mode of action of silver nanoparticles on microbes was not fully known. Inhibition activity of silver nanoparticles against fungi was differing from bacteria because the fungal cell wall greatly differs from the bacterial cell wall. The fungal cell wall is not easily affected by antifungal agents. However, the silver nanoparticle has great antimicrobial activity and biomedical applications. Some of the commercial antibiotics cause bad effect to humans, but silver nanoparticles are considered as good antimicrobial agents with less impediment components in hosts. The possible mechanisms of antifungal action of nanoparticles are cell membrane disruption, cell division inhibition, and cell wall formation inhibition. Fungal cell wall is composed of ergosterol, the silver nanoparticles disrupt the cell membrane by inhibiting ergosterol synthesis or binding with sterol, forming pits and causing the membrane permeability to become leaky leads to cell death $[19,20]$. Silver nanoparticles may affect the mitotic spindle cell division by targeting the microtubule and also inhibit DNA transcription [21]. The silver nanoparticles are positively charged, will interact with negatively charged carboxylic groups and cell wall of mycelia to inhibit the growth of normal budding fungi [22]. Thus, it was concluded that green synthesized silver nanoparticles has a great potential activity against fungi and it is the best platform for deserving further exploration for clinical applications in control diseases.

\section{CONCLUSION}

This present study stated the green-mediated synthesis of silver nanoparticles using the extract of easily available algae $P$. tetrastromatica. The broad peak was observed at $440 \mathrm{~nm}$ for silver nanoparticles. Thus, the synthesized nanoparticles showed cubicaland spherical-structured nanoparticles with agglomeration which was characterized by SEM and TEM. Purity and component of silver nanoparticles were confirmed by EDX. $P$. tetrastromatica extract mediated silver nanoparticles shows high antifungal activity may use as therapeutic agent for future biomedical applications in.

\section{REFERENCES}

1. Singh M, Manikandan S, Kumaraguru AK. Nanoparticles: A new technology with wide applications. Res J Nanosci Nanotechnol 2010;1(1):1-11.

2. Barwal I, Ranjan P, Kateriya S, Yadav SC. Cellular oxido-reductive proteins of Chlamydomonas reinhardtii control the biosynthesis of silver nanoparticles. J Nanobiotechnology 2011;9:56.

3. Frattini A, Pellegri N, Nicastro D, de Sanctis O. Preparation of amine coated silver nanoparticles using triethylenetetramine. Mater Chem Phys 2005;94:148-52.

4. Tao A, Sinsermsuksakul P, Yang P. Polyhedral silver nanocrystals with distinct scattering signatures. Angew Chem Int Ed Engl 2006;45(28):4597-601

5. Mohanpuria P, Rana NK, Yadav SK. Biosynthesis of nanoparticles: Technological concepts and future applications. J Nanopart Res 2008;10(3):507-17.

6. Kaler A, Patel N, Banerjee UC. Green synthesis of silver nanoparticles. Curr Res Inf Pharm Sci 2010;11(4):68-71.

7. Singaravelu G, Arockiamary JS, Kumar VG, Govindaraju K. A novel extracellular synthesis of monodisperse gold nanoparticles using marine alga, Sargassum wightii Greville. Colloids Surf B Biointerfaces 2007;57(1):97-101.

8. Seenivasan R, Rekha M, Indu H, Geetha S. Antibacterial activity and phytochemical analysis of selected seaweeds from Mandapam coast, India. J Appl Pharm Sci 2012;2(10):159-69.

9. Dinesh S, Nasreen N, Jeevan M, Lavanya M, Mythily V, Elanchezhiyan M. Anti hepatitis B virus (HBV) activity of marine brown algae, Padina tetrastromatica. Vet Sci Res 2011;2(2):25-9.

10. Parameswaran PS, Naik CG, Das B, Kamar SY. Constituents of the brown alga Padina tetrastromatica (hauck)-II. Indian J Chem 1996;35:463-7.

11. Sulaiman M, Kurup GM, Rauf AA. Anti inflammatory and antioxidant effect of sulphated polysaccharide isolated from marine algae Padina tetrastromatica from Kerala coast. J Pharm Res 2011;4(3):784-8.

12. Kumar P, Selvi SS, Prabha AL, Kumar KP, Ganeshkumar RS, Govindaraju M. Synthesis of silver nanoparticles from Sargassum tenerrimum and screening phytochemicals for its anti-bacterial activity. Nano Biomed Eng 2012;4(1):12-6.

13. Sosa IO, NoguezC, Barrera RG. Optical properties of metal nanoparticles with arbitrary shapes. J Phys Chem B 2003;107(26):6269-75.

14. Shankar SS, Ahmad A, Sastry M. Geranium leaf assisted biosynthesis of silver nanoparticles. Biotechnol Prog 2003;19(6):1627-31.

15. Jain D, Kumar DH, Kachhwaha S, Kothari SL. Synthesis of plant-mediated silver nanoparticles using papaya fruit extract and evaluation of their antimicrobial activities. Dig J Nanomater Biostruct 2009:4(3):557-63

16. Geoprincy G, Saravanan P, Gandhi NN, Renganathan S. A novel approach for studying the combined antimicrobial effects of silver nanoparticles and antibiotics through agar over layer method and disk diffusion method. Dig J Nanomater Biostruct 2011;6(4):1557-65.

17. Santhoshkumar T, Rahuman AA, Rajakumar G, Marimuthu S, Bagavan A, Jayaseelan C, et al. Synthesis of silver nanoparticles using Nelumbo nucifera leaf extract and its larvicidal activity against malaria and filariasis vectors. Parasitol Res 2011;108(3):693-702.

18. Ahmad N, Sharma S, Alam MK, Singh VN, Shamsi SF, Mehta BR, et al. Rapid synthesis of silver nanoparticles using dried medicinal plant of basil. Colloids Surf B Biointerfaces 2010;81(1):81-6.

19. Sondi I, Salopek-Sondi B. Silver nanoparticles as antimicrobial agent: A case study on E. coli as a model for Gram-negative bacteria. J Colloid Interface Sci 2004;275(1):177-82.

20. Panácek A, Kolár M, Vecerová R, Prucek R, Soukupová J, Krystof V, et al. Antifungal activity of silver nanoparticles against Candida spp. Biomaterials 2009;30(31):6333-40.

21. Rai M, Yadav A, Gade A. Silver nanoparticles as a new generation of antimicrobials. Biotechnol Adv 2009;27(1):76-83.

22. Dibrov P, Dzioba J, Gosink KK, Häse CC. Chemiosmotic mechanism of antimicrobial activity of $\mathrm{Ag}(+)$ in Vibrio cholerae. Antimicrob Agents Chemother 2002;46(8):2668-70. 